\title{
ESTRUTURA DE RELACIONAMENTO ENTRE INSTITUIÇÕES DE PESQUISA DO CAMPO DE CIÊNCIA E TECNOLOGIA NO BRASIL
}

STRUCTURE OF RELATIONSHIP BETWEEN INSTITUTIONS OF RESEARCH OF THE FIELD OF SCIENCE AND TECHNOLOGY IN BRAZIL

\section{RESUMO}

Neste estudo, mapeamos a estrutura de relacionamento entre instituições de pesquisa no campo de Ciência e Tecnologia no Brasil, analisando 688 artigos publicados no Enanpad e no Simpósio de Gestão da Inovação Tecnológica, entre 2000 e 2005. Com base em co-autoria, verificamos que o campo se configura como uma grande rede em que mais da metade das instituições está conectada, criando capital social. Ainda, algumas destacam-se por apresentar grande número de colaboradores, por serem pontes entre diferentes grupos e por manterem a durabilidade das relações, havendo indícios de homofilia, cujas localização geográfica e papel institucional condicionam as relações entre pares. Por fim, existe influência da cooperação na produtividade das instituições.

\section{Luciano Rossoni}

Professor do Programa de Mestrado e Doutorado em Administração, Universidade Positivo

Irossoni@gmail.com

\section{Antônio João Hocayen-da-Silva}

Professor do Curso de Administração e Coordenador dos Cursos de Gestão Financeira e de Marketing, Faculdade de Ensino Superior do Centro do Paraná Ivaiporã.hocayen@yahoo.com.br

\section{Israel Ferreira Júnior}

Doutorando do Centro de Pesquisa e Pós-Graduação em Administração, Universidade Federal do Paraná

isjunior99@yahoo.com.br

Recebido em 21.08.2006. Aprovado em 15.07.2008

Avaliado pelo sistema double blind review

Editor Científico: Rodrigo Bombonati

ABSTRACT In this study we map out the structure of relationships between research institutions in the field of science and technology in Brazil, by analyzing 688 articles published in Enanpad and in the Management Symposium of Technological Innovation, between 2000 and 2005. Based on co-authorship we verified that the field is a great network in which more than half of the institutions are connected, thereby creating social capital. Some institutions also stand out because they have a great number of collaborators, because they form bridges between different groups and because they maintain durable relationships, with indications of homophylia, the geographic locations and institutional role of which condition peer relationships. Finally, the productivity of these institutions is influenced by the cooperation that exists between them.

PALAVRAS-CHAVE Análise de redes sociais, ciência e tecnologia, estrutura de relacionamento, produção científica, redes de pesquisa. KEYWORDS Social network analysis, science and technology, relationship structure, scientific production, research networks. 


\section{INTRODUÇÃO}

Neste estudo, analisa-se a rede social entre instituições brasileiras de ensino e pesquisa da área de Ciência e Tecnologia, a partir de artigos publicados no Enanpad e no Simpósio de Gestão da Inovação Tecnológica no período de 2000 a 2005. Para tanto, foram consideradas as relações de co-autoria entre pesquisadores da área, em que foram identificados 688 artigos que possibilitaram a construção da estrutura de relações.

Buscou-se avaliar as relações entre instituições, pois, apesar do destaque dado na literatura corrente ao processo de inovação em rede no campo de Ciência e Tecnologia, pouco se discutiu sobre a geração de conhecimento nos ambientes em rede no Brasil, e menos ainda sobre a construção do conhecimento científico em redes de pesquisa (MACHADO-DA-SILVA e ROSSONI, 2007).

Diante de tal quadro, pautando-se pela experiência de avaliações em outras áreas do conhecimento, como Física (BARABASI e outros, 2002; GUIMERA e outros, 2005; NEWMAN, 2001a, 2001b, 2001c, 2004), Ciência da Informação (LIU e outros, 2005), Ciências Sociais (MOODY, 2004; WAGNER e LEYDESDORFF, 2005) e a própria Administração (Acedo e outros, 2006; Rossoni e Guarido Filho, 2007), que utilizaram a análise de redes sociais para elucidar a estrutura de um campo científico, almeja-se no presente estudo entender como as instituições de pesquisa no campo da Ciência e Tecnologia buscam colaborar, por meio de seus pesquisadores, para a construção do conhecimento.

Partindo-se do pressuposto de que o conhecimento científico é construído socialmente e influenciado pelos pares que compõem estruturalmente a rede de relações entre as instituições, pretende-se não somente descrever tais relacionamentos, mas também entender como tal estrutura afeta a produção do conhecimento. Já que caracteristicamente as pesquisas em redes sociais buscam avaliar a estrutura de relacionamento entre atores sociais (MIZRUCHI, 2006), este estudo, particularmente, pretende avaliar a estrutura de relações entre as instituições no campo da Administração em Ciência e Tecnologia em três sentidos. O primeiro deles consiste em descrever se o campo realmente se configura como uma rede e qual a centralidade de cada instituição, buscando identificar os grupos, o grau de coesão e o grau de resistência dessa rede. Depois, verifica-se como a posição das instituições em termos regionais e em relação ao seu papel institucional no campo influencia o estabelecimento de relações com outras instituições. Por fim, busca-se verificar se a cooperação influencia a produtividade das instituições em termos de autorias.
Para tanto, este estudo divide-se em cinco seções. Após breve introdução, em que consta o objetivo do estudo, são apresentados os conceitos teóricos relacionados à análise de redes sociais que nortearam a pesquisa. Posteriormente, são discutidos os procedimentos metodológicos e analíticos que sustentaram a análise dos dados. Por fim, são apresentados os resultados e sua discussão, seguidos das considerações finais do estudo.

\section{ANÁLISE DE REDES SOCIAIS}

Emirbayer e Goodwin (1994) salientam que a análise de redes sociais não é somente uma teoria formal ou unitária, mas ampla estratégia de investigação de estruturas sociais. Nelson (1984) afirma que, em termos intuitivos, as redes sociais são conjuntos de contatos que ligam vários atores e que podem ser de diferentes tipos, apresentando conteúdos distintos, bem como diversas propriedades estruturais. A análise de redes sociais é inerentemente de natureza interdisciplinar, possuindo contribuições de áreas como matemática, estatística e computação no ímpeto de produzir aplicações para o método (FREEMAN, 1984).

Existe uma série de conceitos-chave essenciais para a discussão da análise de redes sociais, que são: i) ator entidade (indivíduos, organizações ou países) objeto de estudo na análise de redes sociais; ii) laço relacional - ligação estabelecida entre um par de atores; iii) díade - ligação ou relacionamento estabelecido entre dois atores; iv) tríade - conjunto de três atores e os possíveis laços entre eles; v) subgrupo - conjunto de atores e todos os laços entre eles; vi) grupo - conjunto finito de atores definidos por critérios conceituais, teóricos ou empíricos em que as medidas da rede são tomadas; vii) relação coleção de laços de um tipo específico entre membros de um grupo; e viii) rede social - conjunto finito de atores e as relações entre eles (WASSERMAN e FAUST, 1994; WELLMAN, 1988).

Wasserman e Faust (1994) definem que os métodos relacionados à análise de redes podem ser agrupados em: i) propriedades estruturais, como as medidas de centralidade, densidade, transitividade e coesão; ii) papéis e posições, como a análise de equivalência estrutural, regular e local, e a análise de clusters e de blockmodels; e iii) análise estatística dos relacionamentos, usada para testar proposições teóricas acerca das propriedades relacionais. Para efeitos deste trabalho, serão apresentados aqueles métodos diretamente envolvidos na análise das relações entre instituições de pesquisa no campo da Ciência e Tecnologia no Brasil. 


\section{Propriedades estruturais}

A abordagem relacional focaliza as conexões diretas e indiretas entre os atores (EMIRBAYER e GOODWIN, 1994), buscando entender comportamentos e processos por meio da conectividade entre os atores. Para tanto, são utilizados principalmente dois grupos de medidas: i) centralidade e prestígio, e ii) coesão social.

\section{Centralidade}

Um ator será localmente central se apresentar grande número de conexões com outros atores, e globalmente central se tiver uma posição significantemente estratégica na rede como um todo (SCOTT, 2000). Três medidas são mais comumente utilizadas para avaliar a centralidade dos atores em uma rede: i) centralidade de grau (degree); ii) centralidade de proximidade (closeness); e iii) centralidade de intermediação (betweenness) (HANNEMAN, 2001; HANNEMAN e RIDDLE, 2005; SCOTT, 2000; WASSERMAN e FAUST, 1994).

A centralidade de grau é medida pelo número de laços que um ator possui com outros atores em uma rede (WASSERMAN e FAUST, 1994). Como a centralidade de grau leva em conta somente os relacionamentos adjacentes, segundo Scott (2000), tal medida revela somente a centralidade local dos atores.

A centralidade de proximidade é baseada na proximidade ou distância de um ator em relação aos outros atores em uma rede. A medida de centralidade de proximidade (closeness) de um ator é obtida por meio da soma das distâncias geodésicas entre todos os outros atores (HANNEMAN, 2001; HANNEMAN e RIDDLE, 2005; SCOTT, 2000; WASSERMAN e FAUST, 1994). Segundo Scott (2000), a medida de centralidade de proximidade é indicada para conhecer a centralidade global dos atores.

$\mathrm{Na}$ centralidade de intermediação, a interação dos atores não adjacentes pode depender de outros atores, que potencialmente terão algum controle sobre as interações dos dois atores não adjacentes. Nesse sentido, de acordo com Freeman (1979) e Wasserman e Faust (1994), um ator será intermediário se ligar vários outros atores que não se conectem diretamente.

\section{Coesão social}

Um dos maiores interesses em análise de rede sociais é identificar subgrupos coesos de atores em uma rede. Em termos estruturais, subgrupos coesos são subconjuntos de atores que apresentam laços relativamente fortes, diretos, coesos, intensos e freqüentes (WASSERMAN e FAUST, 1994). Argumenta-se que os subgrupos coesos possuem suas próprias normas, valores, orientações e subculturas (HANNEMAN, 2001; HANNEMAN e RIDDLE, 2005; SCOTT, 2000), sendo base para a solidariedade, identidade e comportamento coletivo mais entre os atores de dentro do grupo do que entre os de fora. Esse fenômeno é conhecido como homofilia (DE NOOY, MRVAR, e BATAGELJ, 2005).

A noção de subgrupo é formalizada a partir da idéia de coesão entre os membros, com base em propriedades específicas de seus laços. Desde que tais propriedades possam ser quantificadas, os subgrupos coesos podem ser formalizados, expondo as diferentes propriedades dos laços (WASSERMAN e FAUST, 1994). Assim, há diferentes modelos teóricos, e Wasserman e Faust (1994) afirmam que as idéias conceitualizadas em subgrupos apresentam quatro propriedades gerais que influenciam a formalização desse conceito: i) mutualidade dos laços; ii) proximidade e alcance entre os membros dos subgrupos; iii) freqüência dos laços entre os membros; e iv) frequência relativa dos laços entre os membros fora e dentro dos subgrupos. Neste trabalho, somente a análise da coesão em termos da mutualidade e da proximidade será usada.

As medidas de subgrupos baseados na mutualidade atêm-se em verificar até que ponto todas as escolhas de pessoas em um subgrupo são mútuas, formando cliques entre os atores. O clique, conceito fundamental para estudar a coesão de subgrupos, é formalmente definido como uma sub-rede ou subgrafo completo de três ou mais nós, em que todos os pontos estejam diretamente conectados (DE NOOY, MRVAR e BATAGELJ, 2005; SCOTT, 2000; WASSERMAN e FAUST, 1994).

A alcançabilidade é uma idéia de subgrupo alternativa que leva em consideração os intermediários do processo social. Para tanto, subgrupos coesos baseados na alcançabilidade requerem que a distância geodésica entre os pontos seja pequena e são formalizados no conceito de $n$-clique. Um n-clique é um subgrupo em que a maior distância geodésica entre dois nós é menor ou igual a $n$ (WASSERMAN e FAUST, 1994), no qual $n$ é o caminho máximo em que membros de um clique podem estar conectados (SCOTT, 2000).

Além das propriedades de formalização dos conceitos acima, há uma abordagem alternativa para identificar os pontos vulneráveis da rede. Basicamente, ela consiste em identificar aqueles nós que, se forem removidos, deixam a estrutura da rede dividida em partes não conectadas (HANNEMAN e RIDDLE, 2005). Tais nós são denominados cut-points. Sua presença em grande quantidade torna as redes mais vulneráveis à fragmentação, pois com a 
remoção desses poucos nós a rede pode ficar desconexa (LI-CHUN e outros, 2006).

\section{Papéis e posições}

De acordo com Wasserman e Faust (1994), "posição" se refere à coleção de indivíduos que estão similarmente imersos em redes de relações, ao mesmo tempo que "papel" se refere às características das relações obtidas entre atores e posições. Para se definir a posição que um ator apresenta num sistema de relações, pode-se recorrer às suas características individuais (atributos), como sexo, grupo social, faixa etária; ou à análise de seus laços, buscando verificar se eles apresentam relações similares às de outros atores (EMIRBAYER e GOODWIN, 1994). No primeiro caso, os indivíduos serão agrupados em uma mesma posição se apresentarem características em comum. Já no segundo, agrupam-se os indivíduos na mesma posição se apresentarem padrões de relações similares.

Existem dois aspectos-chave na análise de papéis e posições: i) identificar as posições sociais como coleções de atores que são similares em seus laços; e ii) modelar os papéis sociais como sistemas de laços entre atores ou entre posições. O primeiro aspecto foca a avaliação de posições a partir de grupos de atores, na qual se utiliza a abordagem de equivalência estrutural (LORRAIN e WHITE, 1971). O segundo foca a avaliação de papéis a partir de grupos de relações, contando com métodos de blockmodels (WHITE, BOORMAN e BREIGER, 1976) e de álgebra relacional (BOORMAN e WHITE, 1976).

\section{Equivalência estrutural}

Dois atores são estruturalmente equivalentes quando têm idênticos laços com outros atores em uma rede (SCOTT, 2000; WASSERMAN e FAUST, 1994). Embora a equivalência estrutural seja utilizada na realização de análise de posições, para Wasserman e Faust (1994) uma completa análise posicional requer quatro passos: i) definição formal de equivalência; ii) medida de equivalência estrutural; iii) representação das equivalências; e iv) avaliação da adequação da representação.

Para elaborar uma posição, é necessário especificar uma definição formal de similaridade entre os atores, utilizando-se para tanto a equivalência estrutural. Em seguida, determina-se a medida de equivalência. Burt (1976) estabelece que a medida de equivalência, baseada na distância euclidiana, avalia se atores $i$ e $j$ apresentam as mesmas entradas nas linhas e colunas das matrizes (HANNEMAN, 2001; HANNEMAN e RIDDLE, 2005; SCOTT, 2000; WASSERMAN e FAUST, 1994). No presen- te estudo, utilizou-se a medida CONCOR (LORRAIN e WHITE, 1971), que é similar à medida de correlação, mas avalia a similaridade a partir dos laços entre os nós.

A representação das posições na rede determina os atores em suas classes equivalentes e as relações entre as classes. O tipo mais comum de representação de classe é o modelo discreto, que fornece a separação dos atores na rede de acordo com as classes equivalentes. Assim, indivíduos estruturalmente equivalentes são condicionados em um número limitado de posições. Finalizando a análise posicional, avalia-se a adequação da representação, que normalmente requer o uso de modelos probabilísticos. Devido à complexidade, essa fase muitas vezes não é realizada, também não sendo desenvolvida neste trabalho.

\section{Blockmodels}

Para White, Boorman e Breiger (1976), blockmodel é um modelo que apresenta as características gerais de uma rede, tanto os laços entre as posições como as informações sobre os atores individuais. Segundo Wasserman e Faust (1994), a técnica se baseia em duas premissas: i) a partição de atores em uma rede é chamada de posições; e ii) para cada par de posições é estabelecida a presença ou ausência de uma relação entre as posições. Wasserman e Faust (1994) afirmam que há três formas de interpretar um blockmodel: i) pela validação do blockmodel feita a partir dos atributos dos atores; ii) a partir da descrição das posições individuais; e iii) a partir da descrição do blockmodel como um todo.

Dependendo da orientação teórica, pode-se afirmar que as características dos atores são importantes condicionantes de suas relações, observadas na estrutura posicional. Por outro lado, a posição estrutural em uma rede pode ser vista como influenciadora das características dos atores. Neste estudo, tais características foram avaliadas em termos da posição geográfica das instituições acerca da unidade federativa em que estão localizadas, o que permite avaliar se elas apresentam certo grau de homofilia - maior intensidade de laços entre instituições do mesmo estado (MCPHEARSON, SMITH-LOVIN e COOK, 2001), ou heterofilia - tendência de relacionamento com instituições de outros estados. Também foram avaliadas as relações entre instituições em referência ao seu papel no campo da pesquisa, definidas tradicionalmente na literatura sobre triple helix como relações entre governo, universidade e empresa (LEYDESDORFF e ETZKOWITZ, 1998). Recorreu-se à idéia de triple helix como definição institucional dos agentes, em que cada esfera - acadêmica, industrial e governamental - tem diferente papel na construção do conhecimento. No entanto, à idéia discu- 
tida por Leydesdorff e Etzkowitz (1998), adicionou-se uma quarta categoria: as agências de fomento governamental, pois estão presentes na estrutura do campo de pesquisa em Ciência e Tecnologia no país.

Avaliando-se o grau de entrada e de saída entre essas posições, pode-se verificar se elas apresentam algum tipo de comportamento, como, por exemplo, se mais transmitem relações do que recebem e vice-versa, ou se estão isoladas. Entender a estrutura de relações entre as posições contribui para a compreensão do comportamento de atores que estão em uma mesma posição social. Por fim, a terceira forma de avaliar um blockmodel consiste em considerar toda a configuração das relações entre as posições, expressa pela matriz imagem. Dessa matriz, pode-se extrair uma rede (visão global) cujos laços representam as relações entre as posições (papéis), úteis para a representação de macroestruturas (SCOTT, 2000).

\section{Análise estatística de dados relacionais: avaliação da influência da cooperação na produtividade}

De acordo com Hanneman e Riddle (2005), uma das principais razões para a utilização de métodos estatísticos em análise de redes é a possibilidade de descrever e entender os padrões de comportamento tanto da rede como um todo quanto de seus atores. Uma das maiores finalidades dessa análise é explicar as relações entre os atores na rede e suas características.

Para os fins deste estudo, será avaliada a relação entre cooperação e produtividade, já que se pode pressupor que instituições com maior capacidade de estabelecer relacionamentos de colaboração apresentam maior potencial de influência sobre o campo, seja por meio de sua produtividade, seja a partir da influência das perspectivas, conceitos, idéias ou metodologias adotadas (MOODY, 2004). Diferentes estudos, conforme relatam Katz e Martin (1997) e Melin (2000), apresentam uma correlação entre cooperação e produtividade. Porém, Lee e Bozeman (2005) advertem que o pressuposto de que a cooperação implica maior produtividade ainda requer maior investigação. De modo similar a Rossoni e Guarido Filho (2007), que avaliaram essa relação no campo da pesquisa em organizações e estratégia, buscou-se avançar no entendimento dessa relação, mas no que se refere à produtividade das instituições do campo de pesquisa em Ciência e Tecnologia.

A partir das definições formais das medidas e técnicas de análise de redes apresentadas neste referencial teórico, que servirá como apoio na interpretação dos dados do presente estudo, discutem-se, logo em se- guida, os procedimentos metodológicos referentes à coleta, tabulação e análise dos dados que dão suporte a este estudo.

\section{DADOS E PROCEDIMENTOS METODOLÓGICOS}

Por meio de pesquisa documental, avaliaram-se todos os artigos publicados nos anais do Enanpad entre os anos de 2000 e 2005, verificando-se 162 artigos, e do Simpósio de Gestão da Inovação Tecnológica, compreendendo as edições de 2000, 2002 e 2004, em que se identificaram 526 artigos. Com um total de 688 artigos, buscou-se identificar a estrutura de relações entre as instituições de ensino e pesquisa em Ciência e Tecnologia por meio de seus laços de co-autoria, empregando-se o método de análise de redes sociais, pois ele possibilita avaliar tanto os aspectos descritivos dos relacionamentos quanto as análises estatísticas causais de tais fenômenos (HANNEMAN, 2001; SCOTT, 2000; WASSERMAN e FAUST, 1994; WELLMAN, 1988).

\section{Tabulação dos dados}

Os dados foram tabulados com o uso do software Microsoft Excel®. Para efeitos de tabulação dos dados, duas instituições são consideradas conectadas se algum dos seus pesquisadores produziu algum artigo conjuntamente, ou seja, são co-autores de algum estudo. Tal procedimento foi utilizado para construir a estrutura de relações entre instituições de pesquisa em Ciência e Tecnologia, que foi representada por meio de uma matriz quadrada cujas linhas e colunas representam as instituições e cujas células indicam a presença ou ausência de relação. Células com valor zero indicam ausência de relação entre instituições, e com valor maior ou igual a 1 indicam relação entre elas.

\section{Métodos de análise dos dados}

A análise de redes foi realizada por meio dos softwares Ucinet 6.0 e Pajek 1.10. Em relação às propriedades estruturais, foram avaliadas a estrutura da rede e seus componentes, as medidas de centralidade e as medidas de coesão, a partir de cliques, n-cliques e cut-points. Para as medidas posicionais da rede, utilizaram-se a análise de equivalência estrutural por meio do algoritmo CONCOR (LORRAIN e WHITE, 1971) e o blockmodeling (DE NOOY, MRVAR e BATAGELJ, 2005). Essa técnica possibilitou representar não somente as relações entre as instituições, mas as relações agrupadas em unidades federativas e entre tipos de instituições (de ensino e 
pesquisa, governo, empresa e agência de fomento governamental), sendo possível verificar a presença e a freqüência de relação entre elas.

Para a avaliação da relação entre os indicadores de centralidade da rede e os indicadores de produção científica, utilizou-se o software SPSS 13®. Foram selecionadas somente as 40 instituições mais prolíficas da área, identificadas por Rossoni, Ferreira Júnior e Hocayen-da-Silva (2006), com o objetivo de reduzir a variância dos dados em relação à centralidade e à produção. Primeiro, criouse a matriz de correlação com todas as variáveis, na qual se verificou a correlação entre as variáveis de produção científica e as variáveis de centralidade. Buscou-se também verificar se alguma das variáveis apresentava multicolinearidade. Depois de as variáveis significativas terem sido identificadas, construíram-se modelos explicativos a partir de regressão linear. Aceitaram-se como significantes os resultados que apresentaram valor de $p<0,05$.

\section{ANÁLISE E DISCUSSÃO DOS RESULTADOS}

Na Figura 1, pode-se observar a estrutura de relações entre as instituições de pesquisa na área de Ciência e Tecnologia. As instituições são representadas pelos nós, e as relações entre eles, por linhas. Instituições que cooperaram apresentam linhas que ligam seus nós correspondentes. Assim, instituições que cooperaram similarmente com outras se apresentam próximas na rede. Como cada linha representa uma relação, instituições com maior colaboração apresentam mais linhas. Para dar destaque a essas instituições, os nós que as representam têm tamanho proporcional ao número de relações. Identifica-se também na rede que nem todas as instituições estão diretamente conectadas, formando diversos componentes isolados uns dos outros. Além disso, para facilitar a visualização da figura, foram excluídas as instituições que não cooperaram com nenhuma outra.

Das 232 instituições de pesquisa, 54 estão isoladas (23\%), ou seja, não cooperaram com nenhuma outra instituição no desenvolvimento da produção científica. Entretanto, apesar do grande percentual de instituições isoladas, $133(57,3 \%)$ estão interligadas diretamente ou indiretamente, formando o componente principal. Componentes são sub-redes cujos nós estão conectados entre si (DE NOOY, MRVAR e BATAGELJ, 2005; HANNEMAN e RIDDLE); os nós pertencentes ao componente de maior tamanho (principal) estão ilustrados em cinza escuro na Figura 1.

Além do componente principal, existem mais 17 componentes que fizeram parte do campo de pesquisa em Ciência e Tecnologia. Porém, como exposto na Tabela 1 , o tamanho dos componentes é bem inferior ao tamanho do componente principal, apresentando grupos de

Figura 1 - Rede de colaboração entre instituições de pesquisa em ciência e tecnologia

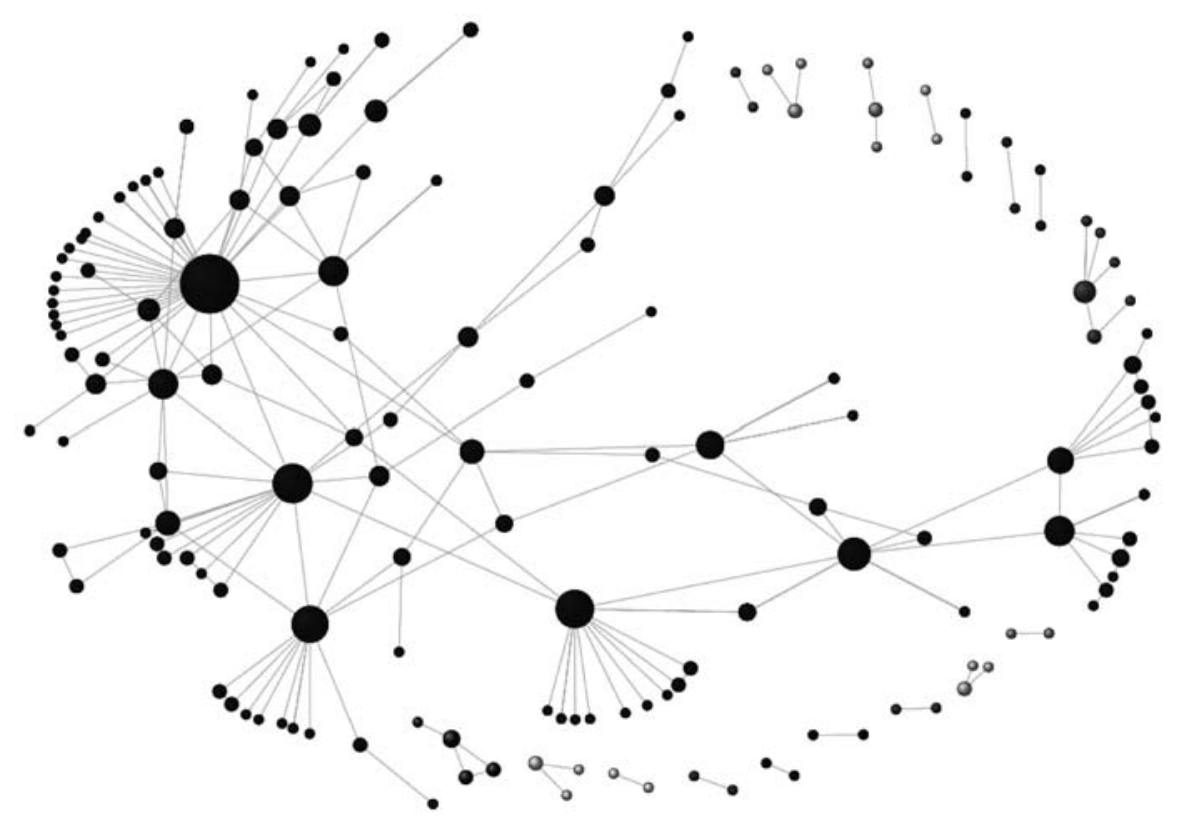


colaboração com 7 instituições, no máximo. Diante de tal quadro, pode-se considerar o campo de pesquisa em Ciência e Tecnologia como relativamente fragmentado, com mais de $40 \%$ das instituições isoladas do maior grupo de colaboração; todavia, existe um grande componente que engloba mais da metade das instituições.

No tocante à configuração estrutural entre as instituições de pesquisa, observa-se que, apesar da baixa densidade da rede $(0,78 \%)$, com pequeno grau de centralização (14,93\%), existe grande número de instituições interligadas, com pequenas distâncias médias entre si $(3,96)$; ou seja, apesar de o campo ter baixa densidade na média, cada instituição, para se relacionar com outra, precisa basicamente de quatro intermediários.

A despeito da baixa densidade geral, o coeficiente de agrupamento entre as instituições é relativamente alto $(0,551)$, o que possibilita que tais instituições tenham proximidade suficiente para a formação de capital social a partir da coesão (COLEMAN, 1990). Além disso, tais organizações apresentam distância média relativamente pequena de todas as outras organizações $(3,96)$, o que possibilita a troca de informações de forma dinâmica dentro do campo. Essa proximidade entre as instituições abre espaço para a troca de experiências entre as diversas instituições, mantendo canal aberto para que a inovação aconteça de forma mais rápida, possibilitando outra maneira de construção do capital social: a partir de laços fracos (GRANOVETTER, 1973) e de buracos estruturais (BURT, 1992). Como já abordado por Kogut e Walker (2001) e Uzzi e Spiro (2005), quando esses dois mecanismos geradores de capital social estão presentes, a estrutura de um campo apresenta uma configuração propícia para a geração de capital social. Tal configuração é conhecida como "small world" ou "pequenos mundos" (WATTS e STROGATZ, 1998). Em pequenos mundos, os atores estão localmente conectados de forma mais coesa. Fora desses grupos, contudo, apresentam laços que interligam globalmente a grande maioria desses atores, o que possibilita a rápida interação dos diversos grupos locais.

Tabela 1 - Distribuição de freqüência entre os componentes

\begin{tabular}{|c|c|c|c|c|}
\hline COMPONENTE & FREQ & FREQ\% & CUMFREQ & CUMFREQ\% \\
\hline Isoladas & 54 & 23.2759 & 54 & 23.2759 \\
\hline 1 & 133 & 57.3276 & 187 & 80.6034 \\
\hline 2 & 2 & 0.8621 & 189 & 81.4655 \\
\hline 3 & 2 & 0.8621 & 191 & 82.3276 \\
\hline 4 & 2 & 0.8621 & 193 & 83.1897 \\
\hline 5 & 3 & 1.2931 & 196 & 84.4828 \\
\hline 6 & 2 & 0.8621 & 198 & 85.3448 \\
\hline 7 & 7 & 3.0172 & 205 & 88.3621 \\
\hline 8 & 2 & 0.8621 & 207 & 89.2241 \\
\hline 9 & 2 & 0.8621 & 209 & 90.0862 \\
\hline 10 & 2 & 0.8621 & 211 & 90.9483 \\
\hline 11 & 4 & 1.7241 & 215 & 92.6724 \\
\hline 12 & 2 & 0.8621 & 217 & 93.5345 \\
\hline 13 & 3 & 1.2931 & 220 & 94.8276 \\
\hline 14 & 3 & 1.2931 & 223 & 96.1207 \\
\hline 15 & 2 & 0.8621 & 225 & 96.9828 \\
\hline 16 & 3 & 1.2931 & 228 & 98.2759 \\
\hline 17 & 2 & 0.8621 & 230 & 99.1379 \\
\hline 18 & 2 & 0.8621 & 232 & 100.0000 \\
\hline Sum & 232 & 100.0000 & & \\
\hline
\end{tabular}


Em relação aos componentes, destaca-se o de maior tamanho, que apresenta as instituições mais prolíficas, como, por exemplo, USP, Unicamp, UFRGS e UFRJ (ROSSONI, FERREIRA JUNIOR e HOCAYEN-DA-SILVA, 2006). Fato que é positivo no campo, pois as instituições que realmente produzem o conhecimento na área não são tão endogênicas, mantendo laços de pesquisa com outras instituições também prolíficas. Dessa forma, faz-se necessário avaliar as medidas individuais das instituições para elucidar melhor o papel desses agentes no campo de pesquisa em Ciência e Tecnologia. Para tanto, algumas propriedades estruturais das instituições de pesquisa serão elucidadas, no caso as medidas de centralidade e de coesão da rede.

No tocante à centralidade de grau, a instituição que manteve o maior número de laços com outras instituições no período foi a USP, como se evidencia na Tabela 2. De todos os laços possíveis, a instituição os manteve com quase $16 \%$ de todas as outras instituições. Além de seu tamanho representativo, ela é um elo entre várias instituições, servindo como suporte para que estas desenvolvam suas pesquisas. Além da USP, a segunda instituição que manteve maior número de colaborações diretas foi a Unicamp, com 16 laços. Apesar de ser a segunda instituição com maior centralidade de grau na rede, ela apresenta menos de metade do número de laços da USP. Ainda assim, demonstra sua importância.

Além da USP e da Unicamp, outras instituições apresentaram significativo número de laços, como, por exemplo, UFRGS, UFRJ e Cefet-PR, com 15, 14 e 11 laços respectivamente. Instituições com grande número de laços representam papel fundamental na estrutura do campo, pois servem como elos principais entre grande parte das instituições, agregando bom número de instituições em sua volta. Das 10 instituições com maior centralidade na rede, somente o IPT-SP não diretamente ligado ao ensino. Todavia, também apresenta programas educacionais, como cursos de mestrado. Diante de tal quadro, podese verificar que as instituições de ensino representam papel fundamental no campo de pesquisa em Ciência e Tecnologia.

A importância de uma instituição na rede não se dá somente pelo número de contatos diretos que mantém, mas também pelas intermediações que realiza. Instituições que são intermediárias de diversas outras instituições podem controlar o fluxo de informação daquelas que estão isoladas, pois são o único ponto de ligação destas com a rede. Dessa forma, estabelecem uma relação de dependência com aquelas que necessitam de suas conexões, pois os benefícios da formação do campo em rede necessariamente perpassam por seus elos. Além disso, servem como ponte para o desencadeamento da informação, sendo mecanismos gerativos de inovação. Como esboçado na Tabela 3, as três instituições com maior centralidade de intermediação - USP, Unicamp e UFRGS - coincidentemente também são as que apresentam maior número de laços (Tabela 2).

Das 10 instituições com maior capacidade de intermediação, todas são instituições de ensino, o que reforça o seu papel como ponte entre os outros tipos de organizações: empresas, governo e agências de fomento. Caso se compare o resultado das 10 instituições com maior número de laços com as 10 maiores intermediárias da rede, pode-se verificar que a UFPR e a Unisinos, apesar de não

Tabela 2 - As 10 instituições com maior número de laços

\begin{tabular}{|c|c|c|c|}
\hline INSTITUIÇÃO & DEGREE & NRMDEGRE & SHARE \\
\hline USP & 36.000 & 15.584 & 0.086 \\
\hline UNICAMP & 16.000 & 6.926 & 0.038 \\
\hline UFRGS & 15.000 & 6.494 & 0.036 \\
\hline UFR & 14.000 & 6.061 & 0.033 \\
\hline CEFET-PR & 11.000 & 4.762 & 0.026 \\
\hline FGV-SP & 9.000 & 3.896 & 0.021 \\
\hline UFSC & 9.000 & 3.896 & 0.021 \\
\hline IPT-SP & 9.000 & 3.896 & 0.021 \\
\hline PUC-PR & 8.000 & 3.463 & 0.019 \\
\hline UFPB & 7.000 & 3.030 & 0.017 \\
\hline
\end{tabular}


apresentarem grande número de laços, têm importante papel como intermediárias.

Além do número de laços que as instituições mantêm, e sua capacidade de intermediação, pode-se verificar o quanto um ator está centralizado globalmente em uma rede, isto é, qual instituição, na média, está mais próxima do conjunto de instituições que fazem parte da rede. Sendo assim, pode-se ver que a instituição mais globalmente centralizada é a Unicamp, pois apresenta a menor distância média entre todas as instituições da rede, conforme Tabela 4. Mesmo apresentando escores bem inferiores aos da USP nas outras medidas de centralidade, a Unicamp se apresenta como o elo mais importante em termos estruturais para a rede como um todo. Já a USP, que apresentou os maiores escores de centralidade de grau e de intermediação, também apresenta alto índice de centralidade global, seguida da UFRGS, que é a terceira instituição mais central nos três índices.

As 10 instituições mais globalmente centrais também estão bem próximas umas das outras (ver círculo central da Figura 2), criando um núcleo pelo qual a informação circula dinamicamente, possibilitando que o conhecimento seja compartilhado em primeira mão entre essas instituições.

Entretanto, apesar de estar visualmente próximas, isso não significa que elas façam parte de um grupo coeso. Para avaliar a coesão de grupos em análise de redes sociais, são úteis algumas medidas, as quais contemplarão as análises seguintes.

As 232 instituições que formaram o campo de produção científica em ciência e tecnologia apresentam 40 cliques de tamanho igual a 3. Desses cliques, a USP participa de 16,

Tabela 3 - As 10 instituições com maior centralidade de intermediação

\begin{tabular}{|c|c|c|}
\hline INSTITUIÇÃO & BETWEENNESS & nBETWEENNESS \\
\hline USP & 4742.117 & 17.851 \\
\hline UNICAMP & 3712.417 & 13.975 \\
\hline UFRGS & 2930.900 & 11.033 \\
\hline CEFET-PR & 2580.300 & 9.713 \\
\hline UFRJ & 1449.400 & 5.456 \\
\hline PUC-PR & 1214.067 & 4.570 \\
\hline UFSC & 1139.000 & 4.288 \\
\hline UFPR & 1070.117 & 4.028 \\
\hline UFPB & 767.000 & 2.887 \\
\hline UNISINOS & 674.083 & 2.537 \\
\hline
\end{tabular}

número bem superior ao das outras instituições. Assim a instituição atua como elo entre os diferentes grupos, conectando-os. Instituições que participam de diferentes grupos têm papel importante na rede, pois tanto integram tais grupos a um grupo maior como servem de mecanismos isomórficos para o estabelecimento de padrões de produção científica.

Porém, se forem avaliados cliques de tamanho igual a 4 , existem somente dois cliques: um formado pelas instituições Cefet-PR, OPET, Senai-PR e UFRGS; o outro formado pelas instituições Centro de Pesquisas Renato Archer, IPTSP, UERJ e Unicamp. Pode-se entender que, no campo de pesquisa em Ciência e Tecnologia, os grupos de pesquisa apresentam pequeno número de instituições envolvidas, algumas atuando localmente, outras com parceiros em estados vizinhos. Isso leva a crer que a colaboração entre as instituições não é prática rotineira, tendo muitas vezes somente o objetivo de realizar uma pesquisa isolada. Tais pesquisas provavelmente não têm continuidade no decorrer dos anos, nem apresentam o envolvimento de novas instituições, o que dificulta a construção do conhecimento na área de forma acumulativa.

Se for avaliada a coesão da rede em relação à alcançabilidade e ao diâmetro dos grupos, pode-se verificar, a partir da avaliação de 2-cliques, que existem dois grupos principais em que a distância máxima entre todas as instituições é de 2 passos. O maior desses grupos, apresentando 37 instituições, tem como instituições mais representativas a USP, a Unicamp e a UFPR. Pode-se afirmar que esse grupo é predominantemente paulista, com pouquíssimas organizações de outros estados. Já o segundo grupo

Tabela 4 - As 10 instituições com maior centralidade de proximidade

\begin{tabular}{|c|c|c|}
\hline INSTITUIÇÃO & FARNESS & nCLOSENESS \\
\hline UNICAMP & 23292.000 & 0.992 \\
\hline USP & 23301.000 & 0.991 \\
\hline UFRGS & 23337.000 & 0.990 \\
\hline IPT-SP & 23348.000 & 0.989 \\
\hline UFPR & 23352.000 & 0.989 \\
\hline UNISINOS & 23360.000 & 0.989 \\
\hline UFRJ & 23372.000 & 0.988 \\
\hline PUC-PR & 23384.000 & 0.988 \\
\hline FINEP & 23386.000 & 0.988 \\
\hline UERJ & 23386.000 & 0.988 \\
\hline
\end{tabular}


apresenta 16 instituições, tendo a Unicamp, a UFRGS e o CEFET-PR como instituições relevantes. Tal grupo é formado principalmente entre instituições do Rio Grande do Sul e do Paraná. Entretanto, como elo entre diferentes grupos desponta a Unicamp, que faz parte tanto do primeiro grupo quanto do segundo. Vale ressaltar que, como em qualquer atividade social, as instituições podem fazer parte de diferentes grupos. Por exemplo, a Unicamp faz parte de dois diferentes grupos na rede de colaboração entre pesquisadores.

Além da avaliação da coesão a partir dos grupos, há 35 instituições que são consideradas cut-points, ou seja, se forem removidas, aumentarão o número de componentes na rede, fragmentando-a, pois são o único elo entre várias instituições. Levando-se em consideração que a rede apresenta 232 instituições, 133 das quais no componente principal, há grande proporção de instituições que mantêm a rede integrada. Todavia, se estas deixarem de desenvolver pesquisas com outras instituições, colaboram para uma maior fragmentação do campo, o que indica que essas redes apresentam alta vulnerabilidade, podendo ser facilmente desintegradas. Como apontam Li-Chun e outros (2006), em redes de colaboração científica, o acúmulo de conhecimento e o senso de identidade dependem da robustez da colaboração. Assim, a aposentadoria, saída ou morte de um pesquisador pode encerrar a conexão entre instituições, levando a rede ao colapso. Diante desse quadro, cabe às instituições buscar, além da colaboração, estabelecer laços com certo grau de redun- dância entre suas parceiras, aumentando a resistência de seus relacionamentos interinstitucionais.

\section{Análise posicional das relações no campo de pesquisa em Ciência e Tecnologia}

Outra tradição em análise de redes sociais que vai além dos elementos estruturais, como a centralidade e a coesão, é a análise posicional das relações. Para avaliar a posição que as instituições ocupam no campo de pesquisa em Ciência e Tecnologia, foi realizada a avaliação de equivalência estrutural das instituições.

A partir do algoritmo CONCOR, verificou-se que existem quatro grupos estruturalmente equivalentes no campo. Como dito anteriormente, o agrupamento das instituições, a partir da equivalência estrutural, é feito de acordo com a similaridade entre os laços que todas as instituições mantêm, criando grupos de acordo com a correlação entre os laços diretos. Um desses grupos é formado por três instituições - Unicamp, USP e UFRJ - que, dentro da rede, apresentam maior quantidade de laços em comum do que com diferentes atores. Outro grupo é formado basicamente por instituições do estado de São Paulo. Já o terceiro é formado basicamente por instituições do Rio Grande do Sul, ficando as demais instituições aglomeradas em outro grupo. Assim, pode-se afirmar que muito da equivalência estrutural entre elas se dá no nível local, pois elas mantêm laços preferencialmente com aquelas do próprio estado. Existe ainda um grupo central, formado por três das quatro instituições mais centrais na rede. Entre essas

Figura 2 - Núcleo da rede entre instituições de pesquisa em ciência e tecnologia

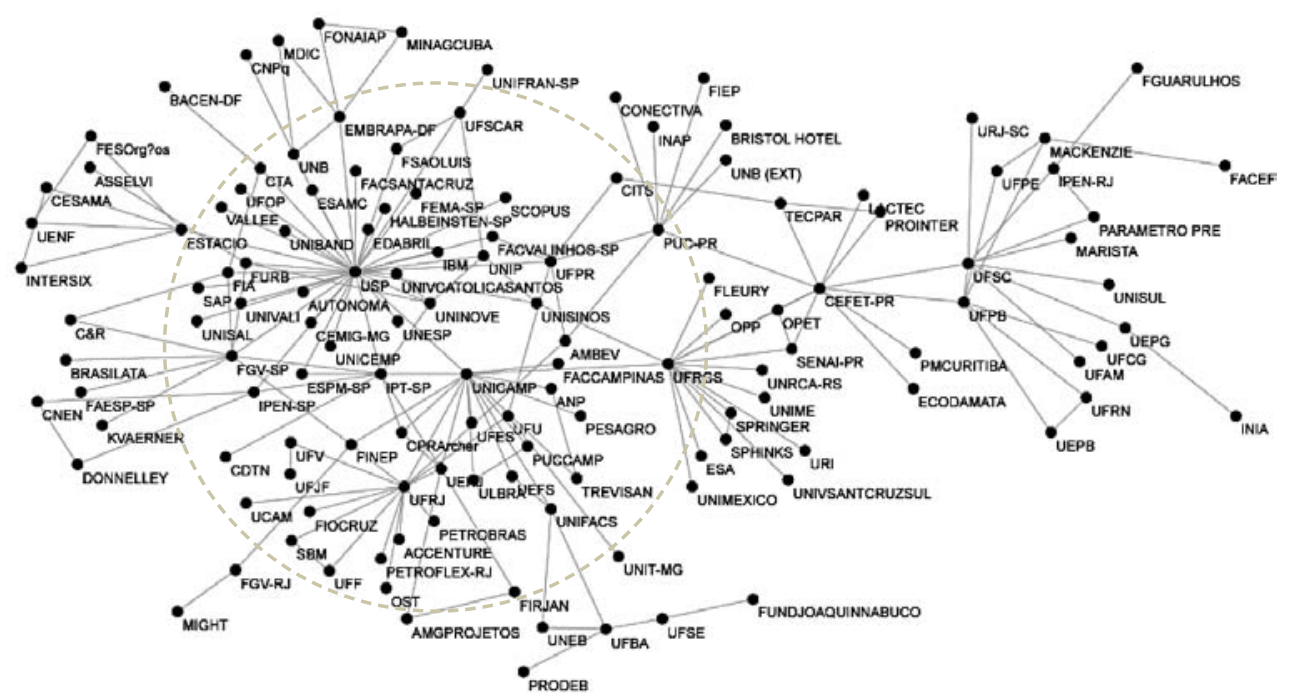


instituições, a informação corre não somente pelos laços diretos, mas também pela equivalência estrutural, ou seja, a partir dos laços indiretos que elas mantêm.

Contudo, diante da presença de um forte componente local, que liga as instituições em termos de referências regionais, buscou-se avaliar como as relações se dão entre os pesquisadores no tocante ao estado federativo das instituições. Para efeitos de análise, foram computados todos os relacionamentos individuais entre pesquisadores, vistos de forma global, identificando-se o padrão de relação entre as unidades federativas.

Como se pode visualizar na Figura 3, os vínculos com pesquisadores filiados às instituições do estado de São Paulo ocorrem em maior freqüência, embora apresentem relações com instituições de 10 estados diferentes. No entanto, os estados do Paraná e Minas Gerais (com sete laços cada), e Santa Catarina e Rio de Janeiro (seis laços cada), também apresentaram vários vínculos com instituições de outros estados. Pode-se verificar que os pesquisadores de estados com menor produção buscam apoio em pesquisadores de instituições de maior porte para desenvolver suas pesquisas, o que leva as instituições centrais a ter papel fundamental na construção do conhecimento no campo de Ciência e Tecnologia.

Se avaliada a proporção de laços que os pesquisadores mantêm com pesquisadores fora do estado em relação ao total de relacionamentos que os pesquisadores localizados nesses estados mantiveram, pode se ver que a colaboração entre pesquisadores da área de Ciência e Tecnologia ocorre primordialmente nos limites da unidade federativa.

De acordo com a Tabela 5, das 17 unidades federativas identificadas no estudo, quatro apresentam mais de $90 \%$ de seus laços entre pesquisadores da própria unidade federativa, e 13, mais de $80 \%$ de seus laços internamente, o que leva a crer, apesar do crescente avanço da tecnologia de informação, que a produção científica na área ocorre primordialmente no nível local. Dessa forma, no campo da pesquisa em Ciência e Tecnologia, as características regionais e a proximidade entre pessoas e organizações atuaram como forte mecanismo de homofilia, em que as relações tendem a ocorrer entre quem está geograficamente próximo.

Estados como SP, RJ, PR e RS, que possuem maior produção científica na área, são algumas das unidades fede-

Figura 3 - Visão global das relações entre instituições de diferentes estados

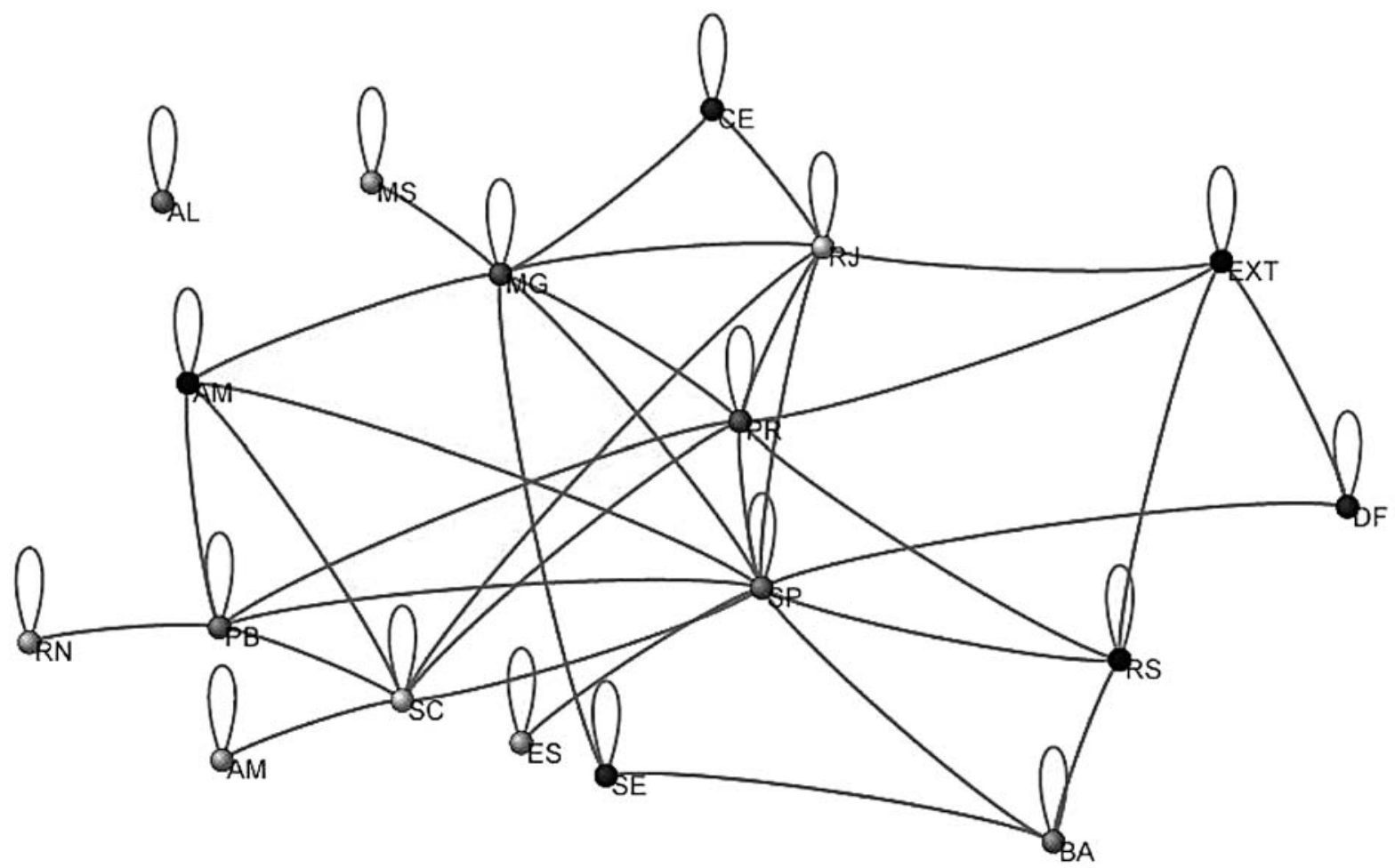


rativas que apresentam maior número de laços recíprocos entre os autores. Outros estados com produção elevada, como MG, BA, SC e o DF, também apresentam elevado número de laços recíprocos. Ao contrário, observa-se que alguns estados com produção mais periférica na área, como, por exemplo, PB, PE, ES, RN, AM, MS e SE, apresentam percentualmente maior número de laços entre pesquisadores de fora do que os estados mais centrais. Tal fato indica que os pesquisadores localizados em estados que não apresentam produção representativa buscam relacionar-se mais freqüentemente com pesquisadores de instituições localizadas em estados com maior produção.

Espera-se que o tipo de instituição a que o pesquisador está vinculado, além de avaliar o papel dos pesquisadores em relação à unidade federativa em que se localizam, também interfira nos padrões de relações entre os pesquisadores. Para tanto, as instituições avaliadas no estudo foram classificadas como: governo (GOV); instituição de ensino e pesquisa (IEP); empresa (EMP); e agência de fomento governamental (AFGOV).

De acordo com a Figura 4, pode-se verificar que o relacionamento entre os pesquisadores do mesmo tipo de
Figura 4 - Visão global das relações por tipo de instituição

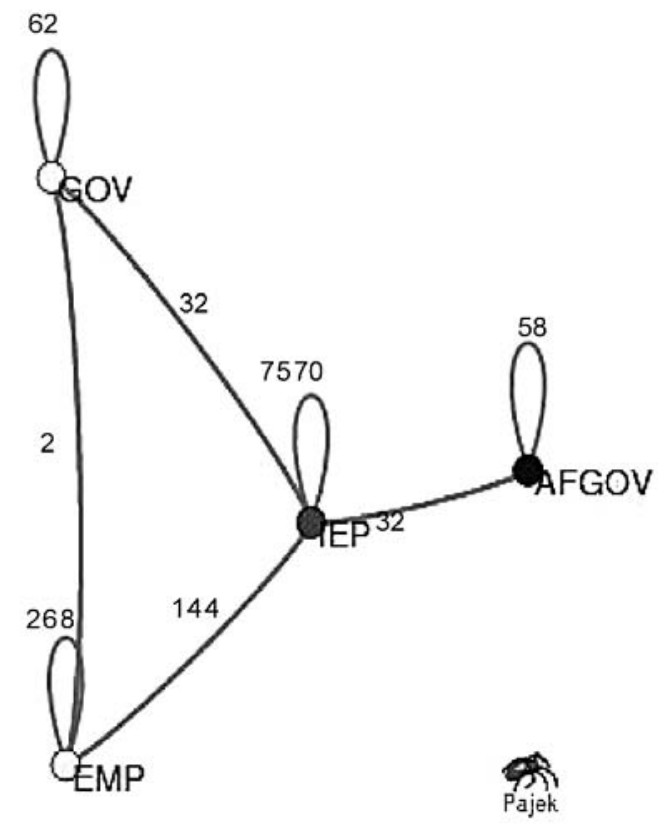

Tabela 5 - Número de laços entre instituições em relação à unidade federativa

\begin{tabular}{|c|c|c|c|c|}
\hline UF & GRAU & LAÇOS REC. & LAÇOS & $\%$ LAÇOS FORA \\
\hline SP & 10 & 1358 & 138 & $9,2 \%$ \\
\hline RJ & 6 & 512 & 92 & $15,2 \%$ \\
\hline PR & 7 & 406 & 54 & $11,7 \%$ \\
\hline RS & 4 & 344 & 30 & $8,0 \%$ \\
\hline DF & 2 & 309 & 30 & $8,8 \%$ \\
\hline MG & 7 & 302 & 46 & $13,2 \%$ \\
\hline $\mathrm{BA}$ & 3 & 199 & 12 & $5,7 \%$ \\
\hline SC & 6 & 191 & 32 & $14,3 \%$ \\
\hline EXT & 4 & 133 & 34 & $20,4 \%$ \\
\hline PB & 5 & 55 & 14 & $20,3 \%$ \\
\hline CE & 2 & 49 & 6 & $10,9 \%$ \\
\hline PE & 4 & 25 & 12 & $32,4 \%$ \\
\hline ES & 1 & 16 & 4 & $20,0 \%$ \\
\hline RN & 1 & 11 & 4 & $26,7 \%$ \\
\hline AM & 1 & 8 & 2 & $20,0 \%$ \\
\hline MS & 1 & 4 & 8 & $66,7 \%$ \\
\hline SE & 2 & 2 & 4 & $66,7 \%$ \\
\hline $\mathrm{AL}$ & 0 & 1 & 0 & $0,0 \%$ \\
\hline
\end{tabular}


instituição é mais freqüente do que com os de outros tipos de instituição (ver valores nos loops - laços em volta dos mesmos nós).

Além disso, pode-se verificar que as agências governamentais de fomento se relacionam diretamente apenas com instituições de ensino e pesquisa. Nesses termos, tais instituições exercem papel fundamental, pois servem como elo entre agências, empresas e governo. O processo de inovação no país é extremamente dependente de instituições de ensino e pesquisa, o que demonstra que as relações entre os diferentes elos da triple helix ocorrem de forma desequilibrada. A necessidade de maior aproximação com os outros elos é emergente, já que a criação de conhecimento é fruto da interação desses diferentes agentes (LEYDESDORFF e ETZKOWITZ, 1998).

\section{Os indicadores de redes têm relação com a produção científica?}

A partir da análise de correlação dos indicadores de centralidade com os indicadores de produção, verificou-se que a centralidade de grau e a centralidade de intermediação apresentam correlação positiva e significativa com a produção $(\mathrm{p}<0,01)$ - correlação de 0,882 para o grau e de 0,861 para a intermediação. Diante da correlação positiva e significante de tais indicadores, foram realizados três modelos de regressão, expostos na Tabela 6 .

O modelo 1 apresenta a relação entre centralidade de grau e produção científica. Segundo esse modelo, o grau corresponde a $86,9 \%$ da explicação da produção científica, o que indica que quanto mais central for uma instituição, mais produtiva ela será. Já o modelo 2 avalia a relação entre centralidade de intermediação e produção científica, explicando $79,3 \%$ da variância em tais indicadores. Contudo, a partir da regressão múltipla (modelo 3), a centralidade de intermediação não se mostrou significativa quando usada em conjunto com a centralidade de grau. Isso ocorreu porque, mesmo individualmente sendo forte e significativa a relação entre intermediação e produtividade das instituições, praticamente toda variância dessa variável é compartilhada com a variável centralidade de grau. Assim, quando colocadas em conjunto no modelo 3 , aquela mais fortemente relacionada com a variável dependente mostrou-se significativa.

Esse resultado reforça que a centralidade de grau apresenta maior poder explicativo na produção científica do que a centralidade de intermediação, o que indica que as instituições com maior número de colaboradores tendem a ser mais produtivas do que as que apresentam baixa cooperação. No entanto, é necessário ressaltar que os dois indicadores mantêm uma relação de dualidade que, em conjunto, contribui tanto para a maior construção de laços quanto para o aumento do número de artigos produzidos por uma instituição. Nesses termos, pode-se afirmar que a posição que uma instituição ocupa na rede de pesquisas do campo de Ciência e Tecnologia apresenta relação direta com sua produção científica.

\section{CONSIDERAÇõES FINAIS}

Objetivou-se com o presente estudo apresentar as propriedades estruturais e posicionais da rede de relações entre as instituições do campo de Ciência e Tecnologia, bem como verificar se tais propriedades apresentam relação com os indicadores de produção científica da área.

Com a análise da rede, verificou-se que mais da metade das instituições estão direta ou indiretamente conectadas, o que contradiz alguns estudos no Brasil, que apontavam para uma grande fragmentação das áreas temáticas. Mesmo a rede apresentando baixa densidade, a distância entre as instituições é pequena, bastando somente quatro intermediários para qualquer contato dentro da rede. Diante do que é apontado pela literatura, a proximidade entre instituições de ensino e pesquisa na área pode fa-

Tabela 6 - Impacto da centralidade na produção científica

\begin{tabular}{|l|c|c|c|}
\hline & MODELO 1 & MODELO 2 & MODELO 3 \\
\hline Grau & $2,263^{*}$ & & $1,861^{*}$ \\
\hline nBetweenness & & $387,526 *$ & 77,46 \\
\hline R & 0,932 & 0,891 & 0,934 \\
\hline$R^{2}$ & $86,9 \%$ & $79,3 \%$ & $87,3 \%$ \\
\hline Sig. & $<0,001$ & $<0,001$ & $<0,001$ \\
\hline${ }^{*} p<0,001$ & $n=40$ & & \\
\hline
\end{tabular}


cilitar a troca de informações, assim como a geração do conhecimento na área, já que elas apresentam uma estrutura de relações propícia para a formação de capital social. Além disso, pode-se observar que três instituições - USP, Unicamp e UFRGS - têm papel extremamente destacado das demais, pois apresentam maior número de colaboradores, o que as coloca em maior proximidade da maioria das outras instituições. Corroborando a importância dessas instituições para o campo de pesquisa em Ciência e Tecnologia, elas também atuam como elo entre os diferentes grupos, contribuindo para a persistência e a durabilidade da rede, já que são os principais cut-points.

No que concerne aos elementos posicionais da rede, verificou-se que a estrutura das relações é fortemente conformada por questões regionais, apresentando a maioria dos laços entre os pesquisadores dentro dos limites dos estados, o que indica alto grau de homofilia. Fato interessante é que as relações, quando ocorrem entre estados diferentes, não se circunscrevem aos estados vizinhos, mas àqueles aparentemente considerados centrais, como São Paulo, Paraná e Minas Gerais. Já no que se refere à avaliação da tríade universidade-indústria-governo, fica claro que a presença em termos de publicações é demasiadamente superior nas instituições de ensino e pesquisa que apresentam ligações com todos os outros tipos de agentes, além de se relacionar exclusivamente com agências de fomento governamental. O mapeamento das relações entre esses diferentes agentes reflete em parte a estrutura de inovação do nosso país, que é dependente de ações governamentais por meio das instituições de ensino e pesquisa.

Verificou-se também que a colaboração entre as instituições está diretamente associada com sua produtividade, pois se encontrou relação forte e significativa entre a centralidade e o número de artigos publicados. Contudo, não se pode afirmar que exista uma relação de causalidade entre estrutura da rede e produção científica, mas uma relação de dualidade. Portanto, constata-se que a construção do conhecimento científico na área de Administração de Ciência e Tecnologia é fortemente influenciada pela estrutura de relacionamento entre pesquisadores e instituições.

Como implicações práticas, este estudo revela que os mecanismos para diminuir a concentração da produção científica na área devem se pautar pelo estímulo ao aumento da cooperação entre programas de pós-graduação mais recentes e de menor tamanho com as instituições de maior representatividade na área. Essas ações poderiam ocorrer por meio do estímulo ao intercâmbio de professores e pela elaboração de programas de fomento que visem à elaboração de pesquisa conjunta entre instituições.
Além dessas ações, grupos de pesquisa cadastrados no CNPq também deveriam privilegiar a cooperação entre programas de diferentes portes. Apesar de a curto prazo tais ações reforçarem ainda mais as instituições de maior tamanho, a longo prazo as instituições posicionadas na periferia se beneficiarão da troca de conhecimento.

Algumas limitações também devem ser apontadas. Por exemplo, não foram consideradas as publicações em periódicos, nem se buscou fazer uma análise longitudinal das relações, o que poderia ter exposto fatos novos. Outros fatores, como tamanho, idade e número de pesquisadores em cada instituição, provavelmente influenciam as relações de cooperação e produtividade, e, por dificuldade na coleta dessas informações, não foram considerados. Poderiam ter sido analisadas, além das relações entre as instituições, as relações entre os pesquisadores, buscando-se verificar se um nível está relacionado com o outro em termos de influência na produtividade. E em vez de analisar apenas as relações de co-autoria, também a cooperação em termos de produção de patentes, licenças ou transferências de tecnologia seria importante para a compreensão do papel das diferentes instituições na estrutura da inovação, para se avaliar seu impacto na inovação.

\section{REFERÊNCIAS}

ACEDO, F; BARROSO, C; CASANUEVA, C; GALÁN, J. Co-authorship in management and organizational studies: an empirical and network analysis Journal of Management Studies, v. 43, n. 5, p. 957-983, 2006.

BARABASI, A; JEONG, H; NÉDA, Z; RAVASZ, E; SCHUBERT, A; VIESEK, T. Evolution of the social network of scientific collaborations. Physica A, v. 311, n. 3-4, p. 590-614, 2002

BOORMAN, S. A; WHITE, H. C. Social structure from multiple networks II. Role structures. American Journal of Sociology, v. 81, n. 6, p. 1384-1446, 1976.

BURT, R. S. Structural Holes: The Social Structure of Competition. Cambridge: Harvard University Press, 1992

BURT, R. S. Position in Networks. Social Forces, v. 55, n. 1, p. 93-122, Sep. 1976.

COLEMAN, J. Foundations of social theory. Chicago: University of Chicago Press, 1990.

DE NOOY, W; MRVAR, A.; BATAGELJ, V. Exploratory social network analysis with pajek. New York: Cambridge University Press, 2005.

EMIRBAYER, M; GOODWIN, J. Network analysis, culture and the problem of agency. American Journal of Sociology, v. 99, n. 6, p. 1411-54, May 1994. 
FREEMAN, L. C. Centrality in social networks: I. Conceptual clarification. Social networks, v. 1, p. 215-239, 1979.

FREEMAN, L. C. Turning a profit from mathematics: the case of social networks. Journal of Mathematical Sociology, v. 10, n. 3-4, p. 343-360, 1984.

GRANOVETTER, M. S. The strength of weak ties. American Journal of Sociology, v. 78, n. 6, p. 1361-1380, 1973.

GUIMERA, R; UZZI, B; SPIRO, J; AMARAL, L. A. N. Team assembly mechanisms determine collaboration network structure and team performance. Science, v. 308, n. 5722, p. 697-702, Apr. 2005.

HANNEMAN, R. A. Introduction to Social Network Methods. Riverside: University of California, 2001.

HANNEMAN, R. A; RIDDLE, M. Introduction to Social Network Methods. Riverside: University of California, 2005. Disponível em: http://faculty.ucr. edu/ hanneman/nettext/index.html. Acesso em 02.05.2006.

KATZ, J. S; MARTIN, B. R. What is research collaboration? Research Policy, v. 26, n. 1, p. 1-18, 1997.

KOGUT, B; WALKER, G. The small world of Germany and the durability of national networks. American Sociological Review, v. 66, n. 3, p. 317-335, Jun. 2001.

LEE, S; BOZEMAN, B. The impact of research collaboration on scientific productivity. Social Studies of Science, v. 35, n. 5, p. 673-702, Oct. 2005.

LEYDESDORFF, L; ETZKOWITZ, H. The triple helix as a model for innovation studies. Science \& Public Policy, v. 25, n. 3. p. 195-2003, 1998.

LI-CHUN, Y; KRETSCHMER, H; HANNEMAN, R. A; ZE-YUAN, L. Connection and stratification in research collaboration: an analysis of the COLLNET network. Information Processing \& Management, v. 42, n. 6, p. 1599-1613, 2006.

LIU, X.; BOLLEN, J; NELSON, M. L; VAN DE SOMPEL, H. Co-authorship networks in the digital library research community. Information Processing E Management, v. 41, n. 6, p. 1462-1480, 2005.

LORRAIN, F; WHITE, H. C. Structural equivalence of individuals in social networks. Journal of Mathematical Sociology, v. 1, n. 1, p. 49-80, 1971.

MACHADO-DA-SILVA, C. L; ROSSONI, L. Persistência e mudança de temas na estruturação do campo científico da estratégia em organizações no Brasil. Revista de Administração Contemporânea, v. 11, n. 4, p. 33-58, 2007.

MCPHEARSON, M; SMITH-LOVIN, L; COOK, J. M. Birds of a feather: homophily in social networks. Annual Review of Sociology, v. 27, p. 415-444, 2001.

MELIN, G. Pragmatism and self-organization: research collaboration on the individual level. Research Policy, v. 29, n. 1, p. 31-40, 2000.

MIZRUCHI, M. S. Análise de redes sociais: avanços recentes e controvérsias atuais. RAE-revista de administração de empresas, v. 46, n. 3, p. $72-86,2006$
MOODY, J. The structure of a social science collaboration network: disciplinary cohesion from 1963 to 1999. American Sociological Review, v. 69, n. 2, p. 213-238, Apr. 2004.

NELSON, R. O Uso da Análise de Redes Sociais no Estudo das Estruturas Organizacionais. RAE-revista de administração de empresas, v. 24, n. 4, p. 150-157, 1984.

NEWMAN, M. E. J. Co-authorship networks and patterns of scientific collaboration. Proceedings of the National Academic Sciences, v. 101, suppl. 1, p. 5200-5205, 2004.

NEWMAN, M. E. J. Scientific collaboration networks. I. Network construction and fundamental results. Physical Review E, v. 64, n. 16131, p. $1-8,2001 \mathrm{a}$.

NEWMAN, M. E. J. Scientific collaboration networks. II. Shortest paths, weighted networks and centrality. Physical Review E, v. 64, n. 16132, p. $1-7,2001 b$.

NEWMAN, M. E. J. The structure of scientific collaboration networks. Proceedings of the National Academic Sciences, v. 98, n. 2, p. 404-409, 2001c.

ROSSONI, L; FERREIRA JÚNIOR, I; HOCAYEN-DA-SILVA, A. J. Administração de ciência e tecnologia: a produção científica brasileira entre 2000 e 2005. In: SIMPÓSIO DE GESTÃO DA INOVAÇÃO TECNOLÓGICA, 24. 2006, Gramado. Anais... Gramado: ANPAD, 2006.

ROSSONI, L; GUARIDO FILHO, E. R. Cooperação interinstitucional no campo da pesquisa em estratégia. RAE-revista de administração de empresas, v. 47, n. 4, p. 74-87, 2007.

SCOTT, J. Social Network Analysis: A Handbook. 2 ed. London: Sage, 2000.

UZZI, B; SPIRO, J. Collaboration and creativity: the small world problem. American Journal of Sociology, v. 111, n. 2, p. 447-504, Sep. 2005.

WAGNER, C. S; LEYDESDORFF, L. Network structure, self-organization, and the growth of international collaboration in science. Research Policy, v. 34, n. 10, p. 1608-1618, 2005.

WASSERMAN, S; FAUST, K. Social Network Analysis: Methods and Applications. Cambridge (MA): Cambridge University Press, 1994.

WATTS, D. J; STROGATZ, S. H. Collective dynamics of "small-world" networks. Nature, v. 393, p. 440-442, Jun. 1998.

WELLMAN, B. Structural analysis: from method and metaphor to theory and substance. In: WELLMAN, B; BERKOWITZ, S. D. Social Structures: A Network Approach. Cambridge (MA): Cambridge University Press, 1988.

WHITE, H. C; BOORMAN, S. A; BREIGER, R. L. Social structure from multiple networks. I. Blockmodel of roles and positions. American Journal of Sociology, v. 81, n. 4, p. 730-779, 1976. 